\title{
An exploration of the feature-positive effect in adult humans
}

\author{
Anja Lotz • Metin Uengoer • Stephan Koenig • \\ John M Pearce $\cdot$ Harald Lachnit
}

Published online: 21 December 2011

(C) Psychonomic Society, Inc. 2011

\begin{abstract}
Experiment 1 compared the acquisition of a feature-positive and a feature-negative discrimination in humans. In the former, an outcome was signaled by two stimuli together, but not by one of these stimuli alone. In the latter, the outcome was signaled by one stimulus alone, but not by two stimuli together. Using a within-group design, the experiment revealed that the feature-positive discrimination was acquired more readily than the feature-negative discrimination. Experiment 2 tested an explanation for these results, based on the Rescorla-Wagner theory, by examining how novel discriminations, based on a combination of a featurepositive and a feature-negative discrimination, were solved. The results did not accord with predictions from the theory. Alternative explanations for the results are considered.
\end{abstract}

Keywords Associative learning · Attention .

Discrimination $\cdot$ Human learning

The similarity between two stimuli is an important influence on the ease with which a discrimination between them can be mastered. Indeed, certain theories of discrimination learning regard this influence as being paramount in determining how rapidly a discrimination will develop (see Pearce, 1994). A clear implication of this basic assumption is that a symmetry should exist between the acquisition of the two possible discriminations between two different patterns - that is, a discrimination between Pattern A, which signals reward, and

A. Lotz $(\bowtie) \cdot$ M. Uengoer $\cdot$ S. Koenig $\cdot$ H. Lachnit Fachbereich Psychologie, Philipps-Universität Marburg,

Gutenbergstrasse 18, 35032 Marburg, Germany

e-mail: lotza@staff.uni-marburg.de

J. M. Pearce

School of Psychology, Cardiff University,

CF10 3AT Cardiff, UK
$\mathrm{B}$, which signals nonreward. $\mathrm{A}+\mathrm{B}-$ should develop just as readily as an $\mathrm{A}-\mathrm{B}+$ discrimination. Although the foregoing claim might not seem contentious, it is contradicted by the feature-positive effect. An early demonstration of this effect was provided by Jenkins and Sainsbury (1969), who required pigeons to peck at one pattern for food, but not at another. Birds that were rewarded for pecking at $\mathrm{AB}$, but not $\mathrm{A}$, solved the discrimination more rapidly than birds rewarded for pecking at $\mathrm{A}$, but not $\mathrm{AB}$. In other words, the feature-positive discrimination, $\mathrm{A}-\mathrm{AB}+$, was acquired more readily than the feature-negative discrimination, $\mathrm{A}+\mathrm{AB}-$. Subsequent experiments have demonstrated a similar feature-positive effect with a variety of species, including humans (e.g., Fiedler, Eckert, \& Poysiak, 1989; Newman, Wolff, \& Hearst, 1980; Richardson \& Massel, 1982).

Several authors have proposed that the feature-positive effect in animals can be understood in terms of the influential Rescorla-Wagner (Rescorla \& Wagner,1972; Wagner \& Rescorla, 1972) theory (Reberg \& LeCLerc, 1977; Soto \& Wasserman, 2010). Since this theory has been used with some success to explain a variety of phenomena of human associative learning, the purpose of the present experiments was to examine whether it can also account for the featurepositive effect in this species. The overall purpose of the investigation was to enhance our understanding of how humans solve discriminations.

According to Rescorla and Wagner (1972), discriminations are solved through the acquisition of associations between the training stimuli and the outcomes they signal. If stimulus $\mathrm{A}$ is paired with reward, there will be an increment in its associative strength, $V_{\mathrm{A}}$, and thus an increment in its capacity to elicit a response. The growth in associative strength is governed by Eq. 1 . In this equation, $\lambda$ is the asymptote for learning set by the magnitude of the reward. On nonrewarded trials, $\lambda$ is zero. $V_{\mathrm{T}}$ is the sum of the associative strengths of all the stimuli present on a trial, 
and $\alpha_{\mathrm{A}}$ and $\beta$ are learning rate parameters set by properties of A and the trial outcome, respectively:

$\Delta V_{\mathrm{A}}=\alpha_{\mathrm{A}} \cdot \beta \cdot\left(\lambda-V_{\mathrm{T}}\right)$.

When applied to a feature-positive discrimination, $\mathrm{A}^{-}$ $\mathrm{AB}+$, the theory predicts that $\mathrm{A}$ and $\mathrm{B}$ will both gain associative strength on trials with the compound, which will be lost by $\mathrm{A}$ on the trials with this stimulus by itself. The discrimination will be solved when $\mathrm{B}$ has asymptotic excitatory strength and $\mathrm{A}$ has no associative strength. In the case of a feature-negative discrimination, $\mathrm{A}+\mathrm{AB}-$, $\mathrm{A}$ will gain associative strength on the trials by itself and will lose it on trials with the compound. The equation also predicts that on the $\mathrm{AB}$ - trials, $\mathrm{B}$ will enter into an association with negative strength — an inhibitory association — which will counter the excitatory effects of A and also protect this stimulus from extinction. At any point during training, the magnitude of the discrimination between $\mathrm{AB}+$ and $\mathrm{A}-$ of the featurepositive discrimination will be determined by the excitatory strength of $\mathrm{B}$, whereas that between $\mathrm{A}+$ and $\mathrm{AB}-$ will be determined by the inhibitory strength of $\mathrm{B}$. Since the theory predicts that the properties of $\mathrm{B}$ will be acquired more rapidly during a feature-positive than during a featurenegative discrimination, it then predicts that the solution of the former will develop more rapidly than the solution of the latter. According to this account, therefore, the featurepositive effect is an inevitable consequence of the mechanisms of discrimination learning.

Newman et al. (1980) reviewed a number of studies with humans that revealed that the feature-positive effect is either weak or absent in participants older than 5 years. (e.g., Norton, Muldrew, \& Strub, 1971; Sainsbury, 1971, 1973). The design of these experiments was relatively simple, and Newman et al. suggested that a performance ceiling made it difficult to reveal the effect. In order to demonstrate a feature-positive effect with humans, their experiments were therefore based on rather more complex discriminations than the $\mathrm{A}+\mathrm{AB}-$ and $\mathrm{A}-\mathrm{AB}+$ designs that characterized earlier studies of this effect with both humans and animals. Participants in one experiment (Experiment 4) were presented with combinations of four different geometric symbols (named A, B, C, and D, hereafter), one of which was the feature (e.g., A). On each trial, three symbols were presented in random positions on a display. Feature trials always contained the symbol $\mathrm{A}$ and two further symbols $(\mathrm{ABC}, \mathrm{ABD}$, and $\mathrm{ACD})$; nonfeature trials always consisted of three symbols other than A (e.g., BCD). Participants were told that there were correct and incorrect displays and that they would earn points for pressing on the correct displays and lose points for pressing on incorrect displays. With this design, Newman et al. found a substantial feature-positive effect in humans. In passing, it can be noted that, using a related design, Jenkins and Sainsbury (1969) were able to demonstrate the same effect with pigeons.

Although the Rescorla-Wagner theory can explain the basic feature-positive effect, it is unable to provide a ready explanation for the results described by Newman et al. (1980). A computer simulation based on Eq. 1 indicated that for the foregoing experiment, the theory predicts that the feature-negative discrimination will be acquired more rapidly than the feature-positive discrimination. The reason behind this prediction is that the nonreinforced trials reduce the associative strength of the individual stimuli to a greater extent in the feature-positive than in the feature-negative discrimination, which results in responding during the reinforced trials being weaker in the former than in the latter. Other successful demonstrations of a feature-positive effect with adult humans have been reported by Richardson and Massel (1982) and Fiedler et al. (1989). However, they too used designs similar to those employed by Newman et al., and their findings are again difficult to explain with the Rescorla-Wagner rule.

It can be argued that these experiments do not provide a fair test of the Rescorla-Wagner theory. As a result of being confronted with three different geometric shapes, participants may have adopted a strategy of focusing on one or two at a time and may have based their prediction of the trial outcome on this sample. The Rescorla-Wagner theory operates on the assumption that all the stimuli on a trial are sampled equally, provided they are of the same salience, and when this does not occur, the outcome of an experiment may deviate from what is predicted theoretically. In order to provide a better test of the analysis offered by the RescorlaWagner theory for the feature-positive effect, each discrimination in the present experiments involved fewer stimuli than in the experiments just described.

\section{Experiment 1}

A single group of participants was given two featurepositive and two feature-negative discriminations, using a predictive learning task. The stimuli were letters presented either individually or in pairs on a computer monitor, with different letters being used for each discrimination. Trials involving a single letter for the feature-negative discrimination and two letters for the feature-positive discrimination were followed by a short presentation of a green circle on the screen. If the participants thought that the circle would follow the letter, or letters, on the screen, they were required to move a cursor over a letter and click a button on a computer mouse. No response was required if it was thought that the circle would not be presented. By using four discriminations and a within-group design, it was hoped that the task would not be so simple that it would suffer from the 
ceiling effects alluded to by Newman et al. (1980) and that the feature-positive discrimination would be acquired more readily than its feature-negative counterpart. As a further step toward encouraging a demonstration of the feature-positive effect, the overall design of the experiment and the instructions given to the participants were based on those used in the successful studies described by Newman et al.

\section{Method}

Participants Thirty-seven students (27 of whom were female) from the Philipps-Universität Marburg were tested individually and needed approximately $20 \mathrm{~min}$ to complete the experiment. Their mean age was 22.30 years (18-36 years). Participants received course credit for participation.

Apparatus and stimuli Participants were tested in a room in the Psychology Department of the university. Instructions and further necessary information were presented on a computer screen. The participants used a computer mouse for responding. The letters E, A, O, Ä, Ö, and $\ddot{U}$ (for the practice trials) and $\mathrm{B}, \mathrm{C}, \mathrm{D}, \mathrm{F}, \mathrm{G}, \mathrm{H}, \mathrm{K}$, and $\mathrm{L}$ (for the experimental trials) were assigned randomly to the different discriminations.

Procedure Participants were told that their task was to predict successfully the occurrence of a green circle on the screen. The following instructions were presented (in German) at the beginning of the experiment:

In this experiment, you will see letters (single or pairs of letters) on the screen. These letters belong to two categories (category 1 and category 2). Whenever a letter or letter pair from category 1 is presented, then subsequently a green circle appears. In contrast, letters or letter pairs from category 2 are never followed by a green circle. Your task is to predict when a letter/letter pair is followed by a green circle. In order to make your prediction, click on the letter or on one of the letters. If your prediction is right (click on a letter/ letter pair from category 1 or no click on category 2 letters), you gain one point. If your prediction is wrong, you do not gain a point. At the end of the experiment, your score will be presented. If your score is high, you will receive an extra reward.

Each trial started with a pause of about $5 \mathrm{~s}$, during which the information "Prepare to start" was presented in the middle of the screen. Then one or two letters (height: approximately $3 \mathrm{~cm}$ ) were presented at a fixed position in the upper half of the screen for about $5 \mathrm{~s}$. Single letters were presented at the center of the screen, pairs of letters were located to the right and left of the midpoint of where individual letters were shown, separated by a gap equivalent to the width of two letters. Instructions to click on one letter if it was thought that the green circle would follow were displayed throughout the experiment. After the letters or letter pairs had disappeared, for about $2 \mathrm{~s}$, either a green circle (diameter, $7.3 \mathrm{~cm}$ ) appeared in the lower half of the screen, or an empty screen was shown. The next trial started after an interval of $5 \mathrm{~s}$.

First, four practice trials were given to make sure that participants understood the procedure. Two practice trials involved one letter displayed on the screen, and the remaining two practice trials involved two letters. One letter and one pair of letters were followed by a green circle, whereas the others were not. The experiment then started, after a short delay.

During the experimental stage, two feature-positive (A$\left.\mathrm{AB}+, \mathrm{C}^{-} \mathrm{CD}+\right)$ and two feature-negative $\left(\mathrm{E}+\mathrm{EF}-, \mathrm{G}^{+} \mathrm{GH}^{-}\right)$ discriminations were given. There were eight blocks of trials, with each block containing the eight different trial types presented just once in a random sequence. There was thus a total of 64 trials in the experiment. At the end of the experiment, the participants were told their scores, and all of them then received a chocolate bar.

Results and discussion

A Type I error rate of $p<.05$ was adopted for all of the statistical tests in this and subsequent experiments. Degrees of freedom were adjusted using the Greenhouse-Geisser correction, when appropriate.

Figure 1 shows the group mean percentages of trials on which the green circle was predicted to occur for trials with and without this outcome for the eight blocks of trials for the two discriminations. By way of example, each point on the plot labeled $\mathrm{FP}+$ represents the mean results for one trial

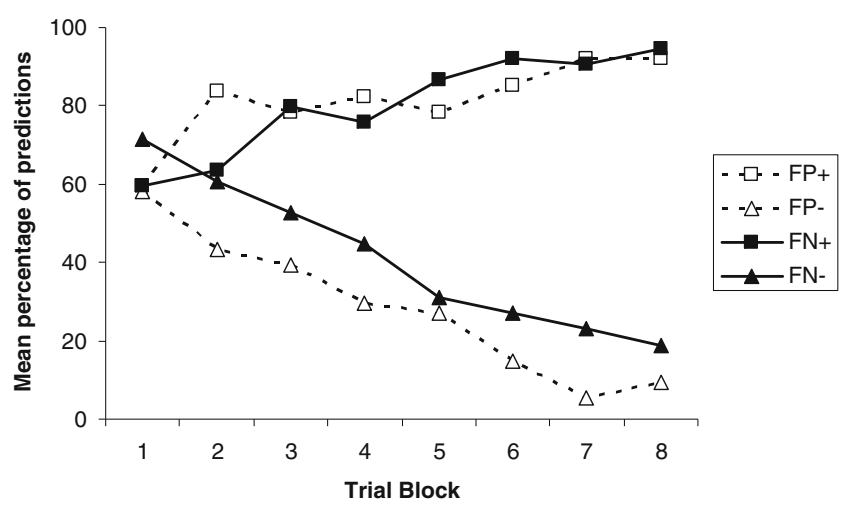

Fig. 1 Mean percentages of predictions that the outcome would occur after the reinforced $(+)$ and nonreinforced $(-)$ trials of the featurepositive (FP) and the feature-negative (FN) discriminations that were given to the single group in Experiment 1 
with each of the reinforced compounds from the two featurepositive discriminations. Before combining the results from each discrimination, we conducted an ANOVA to test whether there were significant differences for the replications of the two kinds of discrimination. Neither the main effect of replication nor any interaction involving this effect was significant, and, for the sake of clarity, the data were therefore pooled. Figure 1 shows that the feature-positive discrimination was acquired more readily than the feature-negative discrimination. In support of this observation, a three-way ANOVA with the factors of discrimination (feature positive or negative), outcome (whether or not the circle was presented on a trial), and trial block revealed a significant main effect of outcome, $F(1,36)=151.36$, discrimination, $F(1,36)=9.64$, and trial block, $F(7,252)=2.87$. There was a significant discrimination $\times$ outcome interaction, $F(1,36)=5.82$, and a significant outcome $\times$ trial block interaction, $F(7,252)=41.48$. The discrimination $\times$ trial block interaction, $F<1$, and the threeway interaction, $F(7,252)=1.93$, were not significant. Tests of simple main effects were conducted to explore the significant discrimination $\times$ outcome interaction. For all the trials combined, the number of trials on which the green circle was predicted to occur did not differ significantly for the two discriminations for trials with a green circle, paired $t(38)<1$, but on trials without a green circle, the outcome was incorrectly predicted to occur more frequently in the feature-negative than in the feature-positive discrimination, $t(38)=3.21$.

The results demonstrate a clear feature-positive effect in which two discriminations of the form $\mathrm{A}-\mathrm{AB}+$ were acquired more readily than two discriminations of the form $\mathrm{A}+$ AB-. Previous demonstrations of this effect with adult humans have used relatively complex discriminations, in which the number of stimuli presented on each trial was three or more (e.g., Newman et al., 1980). Our results show that such complexity is not an essential prerequisite for the feature-positive effect to be observed, since we have demonstrated it with the simplest possible discriminations.

Throughout the experiment, during every compound trial, a record was taken of the letter that was selected. For the eight trial blocks combined, during the compound trials of the feature-positive discrimination, the feature was chosen in preference to the common cue on $62 \%$ of the trials. A one-sample test revealed that this preference was significantly different from chance performance of $50 \%, t(36)=$ 3.43. In contrast, during the trials with the compound for the feature-negative discrimination, the feature was selected on only $39 \%$ of the trials. Once again, this preference was significantly different from chance performance, $t(36)=-2.97$. Moreover, the difference between the results recorded during the compounds from the two discriminations was significant, $t(36)=4.23$.

An outcome similar to that just described was reported by Newman et al. (1980, Experiment 5). Their experiment was much the same as ours, except that a single feature was accompanied by two irrelevant cues on every compound trial and participants were instructed to touch anywhere on the display if they thought that it signaled the outcome of two green lights. Even though the participants received no instruction to select one type of cue over another, they showed a very strong preference for touching the feature in compounds from the feature-positive discrimination and an equally strong preference for not selecting the feature from feature-negative compounds. There is a clear correspondence between these results with humans and those obtained with pigeons. Jenkins and Sainsbury (1969) found that during a feature-positive discrimination, $\mathrm{AX}+\mathrm{A}-$, pigeons pecked more frequently at the feature, $\mathrm{X}$, than at the common cue, A, during compound trials. Conversely during a feature-negative discrimination, $\mathrm{AX}-\mathrm{A}+$, pecks were directed more frequently toward A than toward X. We are not quite sure what to make of this correspondence between the results from such different species. Hearst (1978) attributed the results with pigeons to autoshaping, or sign tracking, which resulted in the birds directing their pecks toward the cue that was most frequently paired with food. To our knowledge, it has not been suggested that the equivalent effect reported by Newman et al. was also due to sign tracking, where the selection or rejection of the feature was due to the influence of Pavlovian conditioned responses reinforced by the trial outcome. Indeed, Newman et al. offered little by way of explanation for their finding. They noted, however, that the tracking responses they recorded were not essential for the emergence of the feature-positive effect, since this effect was found in other experiments in which a preference for making contact with the cue most reliably associated with the outcome was not observed (see their Experiment 4). It is likely, therefore, that the tendency to select one type of cue rather than the other during compound trials was not responsible for the feature-positive effect but was a consequence of processes that led to this effect.

The superior acquisition of the feature-positive over the feature-negative discrimination is in keeping with predictions from the Rescorla-Wagner theory. Figure 2 shows the results from one of a number of computer simulations that were conducted in order to ascertain the outcome predicted by the Rescorla-Wagner theory for Experiment 1 using the simulation program AltSim (Thorwart, Schultheis, König, \& Lachnit, 2009). In order for learning to take place at the rapid rate that was evident, the value of $\alpha$ was set at .5 for all stimuli, and $\beta$ was set at .9 in Eq. 1. The results of the simulation clearly show that the theory predicts a feature-positive effect. Before considering how the results of Experiment 1 might be explained by other theories, we report one further experiment that was designed to evaluate the explanation offered by the Rescorla-Wagner theory for the feature-positive effect. 


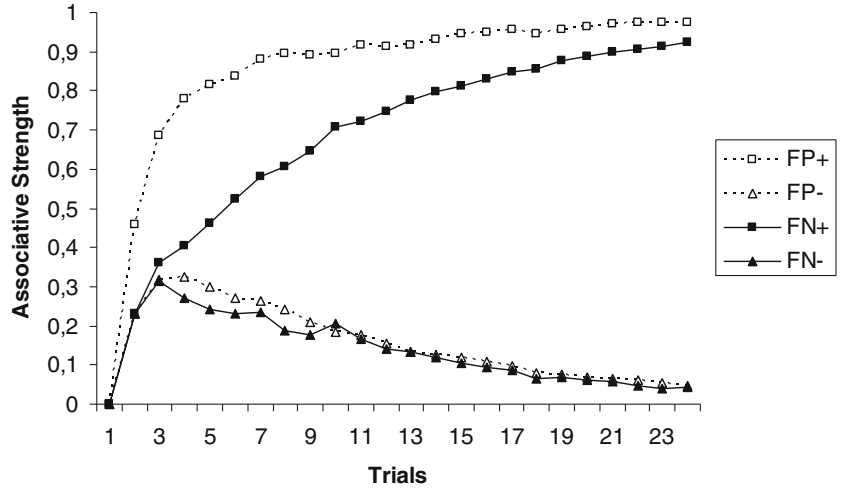

Fig. 2 Associative strength predicted by the Rescorla-Wagner (Rescorla \& Wagner,1972; Wagner \& Rescorla, 1972) theory for the effects of the training in Experiment 1

\section{Experiment 2}

Experiment 2 involved two discriminations, each of which was based on a feature-positive and a feature-negative discrimination. The purpose of the experiment was to test whether the manner in which the discriminations were solved was consistent with predictions derived from the Rescorla-Wagner theory. If the results from the experiment should fail to confirm these predictions, this would imply that the theory provides an inadequate account not only of the feature-positive effect in humans, but also of how humans solve discriminations in general.

One discrimination in the experiment was of the form $\mathrm{A}+$ $\mathrm{AB}-\mathrm{BC}+$, in which stimulus $\mathrm{A}$ and compound $\mathrm{BC}$ were followed by the green circle and $\mathrm{AB}$ was followed by nothing. The trials with $\mathrm{A}+\mathrm{AB}-$ provided the featurenegative component of the discrimination, and the featurepositive component, $\mathrm{B}-\mathrm{BC}+$, was nested within the $\mathrm{AB}-$ $\mathrm{BC}+$ trials. In order to determine the predictions made by the Rescorla-Wagner theory concerning this discrimination, a computer simulation was conducted on the basis of Eq. 1, using the same parameter values as for the previous simulation. The results from the simulation are presented in the left-hand panel of Fig. 3. A striking prediction from the simulation is that the associative strength of the reinforced stimulus $\mathrm{A}$ is predicted to be substantially weaker than that of the nonreinforced compound $\mathrm{AB}$ during the early phase of the discrimination and weaker than that of the reinforced compound $\mathrm{BC}$ for the entire discrimination. If it is accepted that the $\mathrm{AB}-\mathrm{BC}+$ component of the discrimination contains, embedded within it, a feature-positive discrimination, it can be said that the Rescorla-Wagner theory predicts that the feature-positive component of the test discrimination will progress more rapidly than the feature-negative component.

The second discrimination was of the form $\mathrm{D}-\mathrm{DE}+\mathrm{EF}-$, in which trials with $\mathrm{D}-\mathrm{DE}+$ provide the feature-positive component and the feature-negative component, $\mathrm{E}+\mathrm{EF}-$, is embedded within the $\mathrm{DE}+\mathrm{EF}-$ trials. The outcome of a computer simulation for this discrimination, based on the principles just described, can be seen in the right-hand panel of Fig. 3. On this occasion, the theory does not predict a feature-positive effect. Inspection of the figure reveals that the feature-positive discrimination is predicted to be acquired more slowly than the feature-negative discrimination. It is also worth noting that when the predicted results from the two discriminations are compared, the feature-negative component, $\mathrm{A}+\mathrm{AB}^{-}$, of the discrimination shown in the left-hand panel is predicted to be acquired more slowly than the feature-positive component $\mathrm{D}^{-} \mathrm{DE}+$, shown in the righthand panel In other words, a comparison of the effects of training with these subdiscriminations should reveal a profound feature-positive effect.

\section{Method}

Participants, apparatus, and stimuli Twenty-five students from the Philipps-Universität Marburg (14 of whom were female) were tested individually and needed approximately 15 min to complete the experiment. Their mean age was 23.9 years (18-43 years). Participants received course credit for participation. The apparatus and stimuli were the same as for Experiment 1, except that the letters used for the experiment were B, C, D, F, G, and $\mathrm{H}$.

Procedure The procedure was the same as in Experiment 1, except for the nature of the trials presented in the experimental stage. Participants were presented with the six trials from the $\mathrm{A}+\mathrm{AB}-\mathrm{BC}+$ and the $\mathrm{D}-\mathrm{DE}+\mathrm{EF}-$ discriminations. There were eight blocks of trials, with one presentation of each of the six different trial types presented in a
Fig. 3 Associative strength predicted by the Rescorla-Wagner (Rescorla \& Wagner,1972; Wagner \& Rescorla, 1972) theory for the various trials of the two discriminations employed in Experiment 2
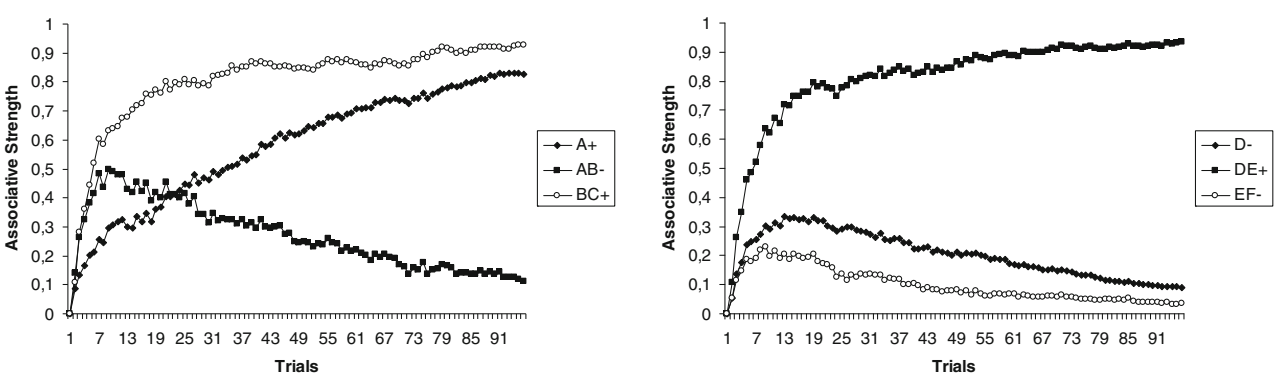
random sequence within each trial block. The instructions to the participants were the same as for Experiment 1. In contrast to Experiment 1, an error in the computer program meant that the letter that was selected on compound trials was not recorded.

\section{Results and discussion}

The results for the $\mathrm{A}+\mathrm{AB}-\mathrm{BC}+$ discrimination are presented in the left-hand panel of Fig. 4. Each trial block represents the mean percentage of trials on which the green circle was predicted to occur for one cycle of the discrimination. In contrast to the predictions derived from the Rescorla-Wagner theory, there was no hint that the green circle was predicted to occur more often on $\mathrm{AB}-$ than on $\mathrm{A}+$ trials during the early stages of training. Indeed, apart from the first trial block, during which performance was similar for both trial types, the opposite was true. There was also no support for the additional prediction that performance during the $\mathrm{A}+$ trials would be inferior to that during $\mathrm{BC}+$ trials. A two-way ANOVA with the factors of trial type $(\mathrm{A}, \mathrm{AB}, \mathrm{BC})$ and trial block revealed a significant main effect of trial type, $F(2,48)=22.99$, but not of trial block, $F(7,168)=1.71$. The trial type $\times$ trial block interaction was also significant, $F(14,336)=2.77$.

Exploration of the significant trial type $\times$ trial block interaction revealed significant main effects of trial type and a significant trial type $\times$ trial block interactions for the $\mathrm{A}+$ and $\mathrm{AB}-$ trials, $F(1,24)=24.60$ and $F(7,168)=3.80$, and for the $\mathrm{BC}+$ and $\mathrm{AB}-$ trials, $F(1,24)=41.22$ and $F(7$, $168)=3.57$, but not for the $\mathrm{A}+$ and $\mathrm{BC}+$ trials, $F_{\mathrm{S}}<1$.

The right-hand panel of Fig. 4 shows that participants again readily discriminated between the trials with and without an outcome during the $\mathrm{D}-\mathrm{DE}+\mathrm{EF}-$ discrimination. A two-way ANOVA, similar to the one just described, revealed a significant main effect of trial type, $F(2,48)=$ 34.10 , and of trial block, $F(1,168)=6.05$, and a significant two-way trial type $\times$ trial block interaction, $F(14,336)=$ 5.20. Further tests involving just two types of trials revealed a significant effect of trial type, $F(1,24)=61.22$, and a significant trial type $\times$ trial block interaction, $F(7,168)=$ 7.69, for the trials with $\mathrm{DE}+$ and $\mathrm{D}^{-}$, while for the pairs of trials $\mathrm{D}^{-}$and $\mathrm{EF}-$, the effect of trial type was significant, $F$ $(1,24)=7.74$, but the interaction was not significant., $F<1$.
For the $\mathrm{DE}+$ and $\mathrm{EF}-$ trials, analyses revealed a significant main effect of trial type, $F(1,24)=30.69$, and a significant trial type $\times$ trial block interaction, $F(7,168)=7.30$.

A comparison of the results from the two discriminations indicates very little difference between the rate at which the feature-positive, $\mathrm{D}-\mathrm{DE}+$, and the featurenegative, $\mathrm{A}+\mathrm{AB}-$, discriminations developed. This observation was supported by the outcome of a three-way ANOVA with the factors of discrimination (feature positive or negative), outcome (whether or not the circle was presented on a trial), and trial block, using the results from the two subdiscriminations. Neither the effect of discrimination, $F(1,24)=3.88$, nor any interaction involving this factor was significant, largest $F(7,168)=2.12$.

Despite their greater complexity, the two discriminations in the present study were solved almost as readily as the simpler discriminations in Experiment 1. In contrast to the results of Experiment 1, however, the outcome of the experiment is not compatible with predictions that were derived from the Rescorla-Wagner theory. A striking prediction of the theory is that the $\mathrm{A}+\mathrm{AB}-$ discrimination, which formed a component of the $\mathrm{A}+\mathrm{AB}-\mathrm{BC}+$ discrimination, would be acquired with considerable difficulty. It was thus predicted that this discrimination would be acquired more slowly than the $\mathrm{AB}-\mathrm{BC}+$ component of the $\mathrm{A}+\mathrm{AB}-\mathrm{BC}+$ discrimination and more slowly than the $\mathrm{D}-\mathrm{DE}+$, feature-positive component of the D- DE+ EF- discrimination. In fact, there was no evidence to support either of these predictions. It might be argued that these failures to confirm predictions of the Rescorla-Wagner theory do not provide a decisive test, because they are essentially null results. A more sensitive task might, perhaps, have revealed findings more consistent with the theory. This argument is challenged by some of the results from the $\mathrm{D}-\mathrm{DE}+\mathrm{EF}-$ discrimination. Inspection of the right-hand panel of Fig. 3 indicates that the theory predicts that the $\mathrm{DE}+\mathrm{EF}-$ discrimination will be acquired more readily than the $\mathrm{D}-\mathrm{DE}+$ discrimination, whereas the opposite was found to be the case. This finding confirms that our method of testing was sufficiently sensitive to reveal significant differences of theoretical importance and that these differences contradict predictions from the RescorlaWagner theory.
Fig. 4 Mean percentages of predictions that the outcome would occur on each of the three trials of the $\mathrm{A}+\mathrm{AB}-\mathrm{BC}+$ (left-hand panel) and the $\mathrm{D}-$ $\mathrm{DE}+\mathrm{EF}-$ (right-hand panel) discriminations in Experiment 2
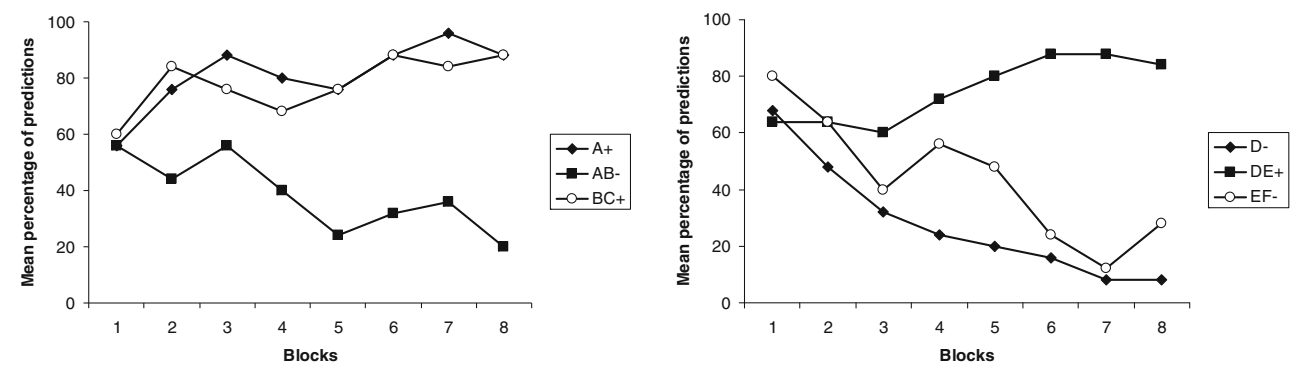


\section{General discussion}

The purpose of the two experiments has been to evaluate the explanation offered by the Rescorla-Wagner theory for the feature-positive effect - that is, for the finding that a featurepositive discrimination, $\mathrm{A}-\mathrm{AB}+$, is acquired more readily than a feature-negative discrimination, $\mathrm{A}+\mathrm{AB}-$. Such an effect was observed in Experiment 1, using a within-group design, and this result is compatible with predictions from the theory. In contrast, the remaining findings that were reported challenge the explanation provided by the Rescorla-Wagner theory for the feature-positive effect. In Experiment 2, a single group received two discriminations, $\mathrm{A}+\mathrm{AB}-\mathrm{BC}+$ and $\mathrm{D}-\mathrm{DE}+\mathrm{EF}-$, which were created by combining a feature-positive and a feature-negative discrimination. In neither discrimination did the pattern of performance accord with that predicted by the theory. A particularly troublesome finding was that the $\mathrm{D}-\mathrm{DE}+$ component of the $\mathrm{D}-\mathrm{DE}+\mathrm{EF}-$ discrimination was acquired more readily than the DE+ EF- component. From the simulation shown in the right-hand panel of Fig. 3, it is evident that the theory predicts the opposite outcome. The present results thus pose a challenge to the explanation offered by the Rescorla-Wagner theory for the feature-positive effect in particular and for discrimination learning in general. What are their implications for other theories of how discriminations are solved?

The Rescorla-Wagner theory is based on the assumption that a training trial with two or more stimuli provides the opportunity for an association to develop between each stimulus and the outcome. According to the theory of Pearce (1987, 1994), only a single association will develop on any trial, which will be between the outcome and the pattern of stimulation in its entirety that precedes the outcome. Moreover, when two patterns of stimulation are paired with different outcomes, the similarity between the patterns will determine the degree of generalization between them and, thus, dictate how readily the discrimination will be solved. It then follows from this analysis that a feature-positive discrimination, $\mathrm{A}-\mathrm{AB}+$, will develop just as readily as a feature-negative discrimination, $\mathrm{A}+\mathrm{AB}-$, based on the same stimuli. The successful demonstration of a featurepositive effect in Experiment 1, as well as demonstrations of this effect in previous experiments (e.g., Newman et al., 1980), clearly challenges this explanation. One way in which the theory of Pearce might overcome this obstacle is to assume that attention falls off to $\mathrm{A}$ in a feature-positive $\mathrm{A}-\mathrm{AB}+$ discrimination because it is irrelevant, whereas attention would be sustained to $\mathrm{A}$ in a feature negative, $\mathrm{A}+\mathrm{AB}-$ discrimination, because the discrimination could not be solved without this stimulus. There is certainly evidence with rats showing that attention to the irrelevant cue declines during the course of a feature-positive discrimination (Haselgrove,
Esber, Pearce, \& Jones, 2010). Such a change in attention during a feature-positive discrimination would then reduce the similarity of the signals for reward and nonreward and would facilitate the acquisition of this discrimination, relative to its feature-negative counterpart. A similar change in attention might be expected to take place with $\mathrm{D}$ in the $\mathrm{D}-\mathrm{DE}+\mathrm{EF}-$ discrimination in Experiment 2 and then allow the theory to predict the pattern of results that was observed on this occasion. In the case of the $\mathrm{A}+\mathrm{AB}-\mathrm{BC}+$ discrimination in the final experiment, all three stimuli were relevant and should continue to be paid full attention. George and Pearce (2011) have extended the configural theory of Pearce (1994) by incorporating into it a mechanism that enhances the salience of stimuli that are relevant to the solution of a discrimination and reduces the salience of those that are irrelevant. They showed, by means of a computer simulation, that this revision enables the theory to predict the feature-positive effect. Additional simulations revealed that the modified theory is also able to predict the correct solution to the discriminations studied in Experiment 2.

We considered the possibility that a similar attentional mechanism would enable the Rescorla-Wagner (Rescorla \& Wagner,1972; Wagner \& Rescorla, 1972) model to account for the results from Experiment 2. The two main problems for the original version of the model are (1) the prediction that the influence of EF will decline more readily than will that of $\mathrm{D}$ in the $\mathrm{D}-\mathrm{DE}+\mathrm{EF}-$ discrimination and (2) the prediction that the influence of A will, for a while, be weaker than that of $\mathrm{AB}$ and, for considerably longer, be weaker than will that of $\mathrm{BC}$ in the $\mathrm{A}+\mathrm{AB}-\mathrm{BC}+$ discrimination. Indeed, allowing the salience of the relevant cues, $\mathrm{E}$ and $\mathrm{F}$, in the $\mathrm{D}-\mathrm{DE}+\mathrm{EF}-$ discrimination to be greater than that of the irrelevant cue, $\mathrm{D}$, allows the model to predict the outcome that we observed. On the other hand, for the $\mathrm{A}+\mathrm{AB}-\mathrm{BC}+$ discrimination, all cues are relevant and should thus be of similar high salience, and the model's predictions do not change. Thus, even when account is taken of attentional processes, at least some of the results of Experiment 2 remain a problem for the Rescorla-Wagner theory.

Instead of being a consequence of the associative mechanisms of learning, Newman et al. (1980; see also Hearst, 1978) argued that the feature-positive effect is a consequence of a strategy individuals adopt when they are required to identify which cues predict an outcome and which do not. They proposed that individuals are more likely to regard a cue as being relevant to the solution of a discrimination if it occurs on trials with, rather than without, an outcome. Thus, for a feature-positive discrimination, $\mathrm{A}-\mathrm{AB}+$, since the relevant cue, $\mathrm{B}$, appears only on reinforced trials, it will be identified rapidly as the cue that signals the outcome will be presented. In contrast, for the feature-negative discrimination, $\mathrm{A}+\mathrm{AB}-$, the same strategy will result in A being selected, incorrectly, as the 
relevant cue. At least during the early stages of training, therefore, these different strategies will hinder the acquisition of the feature-negative discrimination, relative to the feature-positive discrimination. Although this proposal explains the feature-positive effect that was observed in Experiment 1, the results from Experiment 2 are hard to understand from this perspective. As a result of being exposed to the $\mathrm{D}-\mathrm{DE}+\mathrm{EF}-$ discrimination, participants eventually predicted that the green circle would follow the majority of trials with DE and would follow rather few of the trials with $\mathrm{E}$ and EF. However, the tendency to respond that EF would not be followed by a green circle was acquired more slowly than for D. It is hard to see how a strategy of focusing on the cues presented on DE+ trials, but not on the remaining trials, would produce this pattern of results. Participants may well have approached the experiments with a tendency to adopt the strategy suggested by Newman et al., but as this attractively simple proposal is formulated, it is not possible to draw precise predictions from it concerning the course of acquisition of the discriminations in Experiment 2.

The design of the present experiments was based on a series of studies by Newman et al. (1980), which also revealed a successful feature-positive effect. It is conceivable that had we adopted a rather different design, the experiments might not have revealed the same outcome. For instance, Williams, Sagness, and McPhee (1994) have argued that participants will approach a problem with a disposition to treat the combinations of stimuli either elementally or configurally. Moreover, this disposition may be modified by the instructions that are given or the nature of the task (Melchers, Üngör, \& Lachnit, 2005). In the case of Experiment 2, for example, it remains possible that had we used a different task, or a different set of instructions, an outcome that is more in keeping with predictions from the Rescorla-Wagner theory would have been found.

The feature-positive effect can be said to be a robust characteristic of human discrimination learning. It is thus particularly frustrating to discover that no single theory of learning can provide a wholly satisfactory explanation for this effect, unless it is assumed that this effect is a consequence of more attention being paid to relevant than to irrelevant stimuli. Future theoretical success in understanding the basic findings of human associative learning may, therefore, depend on gaining more evidence about the role of changes in attention during discrimination learning. To end on a less speculative note, the reported findings can be regarded as novel in several ways. They have demonstrated the feature-positive effect in humans for the first time with a simple within-group (Experiment 1) methodology.
They have also shown for the first time how humans solve discriminations formed from combining a featurepositive and a feature-negative problem (Experiment 2). Finally, the overall pattern of results from both experimentsmake it unlikely that the feature-positive effect occurs for reasons embodied within the Rescorla-Wagner theory.

Author note The experiments were conducted while John Pearce stayed at the Philipps-University Marburg as a recipient of the Humboldt Award.

\section{References}

Fiedler, K., Eckert, C., \& Poysiak, C. (1989). Asymmetry in human discrimination learning: Feature positive effect or focus of hypothesis effect? Acta Psychologica, 70, 109-127.

George, D. N., \& Pearce, J. M. (2011). A configural theory of attention and associative learning. Manuscript submitted for publication.

Haselgrove, M., Esber, G. R., Pearce, J. M., \& Jones, P. M. (2010). Two kinds of attention in Pavlovian conditioning: Evidence for a hybrid model of learning. Journal of Experimental Psychology. Animal Behavior Processes, 36, 456-470.

Hearst, E. (1978). Stimulus relationships and feature selection learning and behavior. In S. Hulse, H. Fowler, \& W. K. Honig (Eds.), Cognitive processes in animal behavior (pp. 51-88). Hillsdale, NJ: Erlbaum.

Jenkins, H. M., \& Sainsbury, R. S. (1969). The development of stimulus control through differential reinforcement. In N. J. Mackintosh \& W. $\mathrm{K}$. Honig (Eds.), Fundamental issues in associative learning (pp. 123-161). Halifax, NS: Dalhousie University Press.

Melchers, K. G., Üngör, M., \& Lachnit, H. (2005). The experimental task influences cue competition in human causal learning. Journal of Experimental Psychology. Animal Behavior Processes, 31, 477483.

Newman, J., Wolff, W. T., \& Hearst, E. (1980). The feature-positive effect in adult human subjects. Journal of Experimental Psychology. Human Learning and Memory, 6, 630-650.

Norton, G. R., Muldrew, D., \& Strub, H. (1971). Feature-positive effect in children. Psychonomic Science, 23, 317-318.

Pearce, J. M. (1987). A model of stimulus generalization for Pavlovian conditioning. Psychological Review, 94, 61-73.

Pearce, J. M. (1994). Similarity and discrimination: A selective review and a connectionist model. Psychological Review, 101, 587-607.

Reberg, D., \& LeClerc, R. (1977). A feature positive effect in conditioned suppression. Animal Learning and Behavior, 5, 143-147.

Rescorla, R. A., \& Wagner, A. R. (1972). A theory of Pavlovian conditioning: Variations in the effectiveness of reinforcement and nonreinforcement. In A. H. Black \& W. F. Prokasy (Eds.), Classical conditioning II: Current research and theory (pp. 6499). New York: Appleton-Century-Crofts.

Richardson, W. K., \& Massel, H. K. (1982). The feature-positive effect in adult humans: Within-group design. The American Journal of Psychology, 95, 125-138.

Sainsbury, R. S. (1971). The "feature positive effect" and simultaneous discrimination learning. Journal of Experimental Child Psychology, 11, 347-356. 
Sainsbury, R. S. (1973). Discrimination learning utilizing positive or negative cues. Canadian Journal of Psychology, 27, 46-57.

Soto, F. A., \& Wasserman, E. A. (2010). Error-driven learning in visual categorization and object recognition: A common-elements model. Psychological Review, 117, 349-381.

Thorwart, A., Schultheis, H., König, S., \& Lachnit, H. (2009). ALTSim: A MATLAB simulator for current associative learning theories. Behavior Research Methods, 41, 29-34.
Wagner, A. R., \& Rescorla, R. A. (1972). Inhibition in Pavlovian conditioning: Application of a theory. In R. A. Boakes \& M. S. Halliday (Eds.), Inhibition and learning (pp. 301-336). New York: Academic Press.

Williams, D. A., Sagness, K. E., \& McPhee, J. E. (1994). Configural and elemental strategies in predictive learning. Journal of Experimental Psychology. Learning, Memory, and Cognition, 20, 694-709. 